J. Nonlinear Var. Anal. 4 (2020), No. 1, pp. 127-134

Available online at http://jnva.biemdas.com

https://doi.org/10.23952/jnva.4.2020.1.09

\title{
CONTINGENT DERIVATIVES OF THE SET-VALUED SOLUTION MAP OF A NONCOERCIVE SADDLE POINT PROBLEM. A CROSS-FERTILIZATION BETWEEN VARIATIONAL ANALYSIS AND INVERSE PROBLEMS
}

\author{
BAASANSUREN JADAMBA ${ }^{1, *}$, AKHTAR A. KHAN ${ }^{1}$, MIGUEL SAMA $^{2}$, CHRISTIANE TAMMER $^{3}$ \\ ${ }^{1}$ School of Mathematical Sciences, Rochester Institute of Technology, Rochester, New York, 14623, USA \\ ${ }^{2}$ Departamento de Matemática Aplicada, \\ Universidad Nacional de Educación a Distancia, Calle Juan del Rosal, 12, 28040 Madrid, Spain \\ ${ }^{3}$ Institute of Mathematics, Martin-Luther-University of Halle-Wittenberg, \\ Theodor-Lieser-Str. 5, D-06120 Halle-Saale, Germany
}

\begin{abstract}
We focus on the inverse problem of parameter identification in an abstract saddle point problem. Under the assumption that the saddle point problem is solvable, we study the characterizations of the first-order and the second-order contingent derivatives of the set-valued parameter-to-solution map.
\end{abstract}

Keywords. Saddle point problems; Parameter identification; Babuska-Brezzi condition; Elliptic regularization; Output least-squares.

\section{INTRODUCTION}

Let $B$ be a real Banach space and $A$ be a nonempty, closed, convex, and bounded subset of $B$. Let $V$ and $Q$ be real Hilbert spaces with $V^{*}$ and $Q^{*}$ as their dual spaces. We denote the norm of a normed space $N$ by $\|\cdot\|_{N}$ and denote the strong convergence by $\rightarrow$ and the weak convergence $\rightarrow$. Let $a: B \times V \times V \rightarrow \mathbb{R}$ be a trilinear form, which is symmetric in the last two arguments, $b: V \times Q \rightarrow \mathbb{R}$ a bilinear form, $f \in V^{*}$, and $g \in Q^{*}$. Let $a$ be positive and continuous, and let $b$ be continuous, that is, there are positive constants $\kappa_{0}$ and $\varsigma_{0}$ such that

$$
\begin{aligned}
a(\ell, v, v) & \geq 0, \text { for all } \ell \in A, u, v \in V, \\
|b(v, q)| & \leq \kappa_{0}\|v\|_{V}\|q\|_{Q}, \text { for all } v \in V, q \in Q, \\
|a(\ell, u, v)| & \leq \varsigma_{0}\|\ell\|_{B}\|u\|_{V}\|v\|_{V}, \text { for all } \ell \in B, u, v \in V .
\end{aligned}
$$

Consider the saddle point problem: Given $\ell \in A$, find $(u(\ell), p(\ell)):=(u, p) \in V \times Q$ such that

$$
\begin{aligned}
a(\ell, u, v)+b(v, p) & =f(v), \text { for every } v \in V, \\
b(u, q) & =g(q), \text { for every } q \in Q .
\end{aligned}
$$

The primary motivation of this work is to address an important aspect of the inverse problem of identifying a parameter $\ell \in A$ such that the corresponding solution $(u(\ell), p(\ell))$ is closest, in

\footnotetext{
${ }^{*}$ Corresponding author.

E-mail addresses: bxjsma@rit.edu (B. Jadamba), aaksma@rit.edu (A.A. Khan), msama@ind.uned.es (M. Sama), christiane.tammer@mathematik.uni-halle.de (C. Tammer).

Received October 8, 2019; Accepted November 20, 2019.
}

(c)2020 Journal of Nonlinear and Variational Analysis 
some norm, to the given data $(\bar{z}, \hat{z})$, given in some suitable spaces. For this inverse problem to be well-defined the parameter-to-solution map $\ell \mapsto(u(\ell), p(\ell))$ should be nonempty for each $\ell \in A$. Unfortunately, conditions (1.1), for a fixed $\ell \in A$, do not guarantee that (1.2) has a solution. On the other hand, if we additionally require that there are constants $\kappa_{1}>0$ and $\varsigma_{1}>0$ such that

$$
\begin{aligned}
& a(\ell, v, v) \geq \varsigma_{1}\|v\|_{V}^{2}, \quad \text { for all } \ell \in A, v \in L, \\
& \sup _{u \in V} \frac{b(u, q)}{\|u\|_{V}} \geq \kappa_{1}\|q\|_{Q}, \quad \text { for all } q \in Q,
\end{aligned}
$$

where $L:=\{u \in V \mid b(u, q)=0$, for all $q \in Q\}$, then the saddle point problem (1.2) is uniquely solvable. That is, for $\ell \in A$, the parameter-to-solution map $\ell \mapsto(u(\ell), p(\ell))$ is a well-defined and single-valued.

The Babuska-Brezzi or the Inf-Sup condition (1.4), is an extension of the coercivity condition and plays an important role in the study of saddle point problems. However, there are examples when either the Babuska-Brezzi condition does not hold or it is unsatisfactory from a theoretical viewpoint, see $[1,2]$, and the cited references therein.

The objective of this short note is to provide characterizations of the first-order and secondorder contingent derivatives of the parameter-to-solution map. We work under the assumption that the trilinear form $a$ is positive and continuous, the bilinear map $b$ is continuous and that the saddle point problem (1.2) is solvable for every parameter $\ell \in A$. Under this assumption, although the parameter-to-solution map is well-defined, it is a set-valued map, in general. We focus on studying the smoothness of this set-valued map. In the field of variational analysis and set-valued optimization, there are many ways to differentiate set-valued maps (see [9]). Assuming that the parameter-to-solution map admits the first-order and the second-order contingent derivatives, we derive explicit characterizations of these derivatives. These results provide exciting connections between seemingly diverse disciplines, namely, variational analysis, set-valued optimization, and inverse problems.

\section{MAin RESUlts}

In the following, we continue to work under (1.1), but assume additionally that the saddle point problem (1.2) is solvable. Our first objective here is to investigate the intrinsic features of the set-valued parameter-to-solution map. For a given parameter $\ell \in A$, by $\mathscr{U}(\ell)$ we denote the set of all solutions of saddle point problem (1.2). We begin with the following simple result:

Lemma 2.1. For any $\ell \in A$, the solution set $\mathscr{U}(\ell)$ of saddle point problem (1.2) is closed and convex.

Proof. The proof follows at once from the definition of the set-valued map $\mathscr{U}: A \rightrightarrows V \times Q$.

Our goal is to give a derivative characterization for the set-valued parameter-to-solution map. In the literature, a wide variety of derivative concepts have been used to differentiate set-valued maps (see [7]). We will employ the first-order and the second-order contingent derivatives of the set-valued map $\mathscr{U}: A \rightrightarrows V \times Q$. The first-order contingent derivative is defined by using the contingent cone, and the second-order contingent derivative is given by using the second-order contingent set. We recall these notions in the following: 
Definition 2.1. Let $X$ be a normed space, $S \subset X$, and $\bar{z} \in \operatorname{cl}(S)$ (closure of $S$ ). The contingent cone $C(S, \bar{z})$ of $S$ at $\bar{z}$ is the set of all $z \in X$ such that there are sequences $\left\{t_{n}\right\} \subset \mathbb{P}:=\{t \in$ $\mathbb{R} \mid t>0\}$ and $\left\{z_{n}\right\} \subset X$ with $t_{n} \downarrow 0$ and $z_{n} \rightarrow z$ satisfying $\bar{z}+t_{n} z_{n} \in S$, for each $n \in \mathbb{N}$. The second-order contingent set $C^{2}(S, \bar{z}, w)$ of $S$ at $\bar{z} \in \operatorname{cl}(S)$ in the direction $w \in X$ is the set of all $z \in S$ such that there are a sequence $\left\{z_{n}\right\} \subset X$ with $z_{n} \rightarrow z$ and a sequence $\left\{t_{n}\right\} \subset \mathbb{P}$ with $t_{n} \downarrow 0$ satisfying $\bar{z}+t_{n} w+t_{n}^{2} z_{n} / 2 \in S$, for each $n \in \mathbb{N}$.

Remark 2.1. It is known that the contingent cone $C(S, \bar{z})$ is a nonempty closed cone. However, $C^{2}(S, \bar{z}, w)$ is only a closed set (possibly empty), non-connected in general, and it may be nonempty only if $w \in C(S, \bar{z})$. For details of these concepts and their applications, see [7].

Next we collect some notions for set-valued maps. Given normed spaces $X$ and $Y$, let $F$ : $X \rightrightarrows Y$ be a set-valued map. The (effective) domain and the graph of $F$ are defined by

$$
\operatorname{dom}(F):=\{x \in X \mid F(x) \neq \emptyset\}
$$

and

$$
\operatorname{graph}(F):=\{(x, y) \in X \times Y \mid y \in F(x), x \in \operatorname{dom}(F)\} .
$$

We now introduce the first-order and the second-order contingent derivatives.

Definition 2.2. Let $X$ and $Y$ be normed spaces, $F: X \rightrightarrows Y$ be a set-valued map, and $(\bar{x}, \bar{y}) \in$ $\operatorname{graph}(F)$. The contingent derivative of $F$ at $(\bar{x}, \bar{y})$ is the set-valued map $D F(\bar{x}, \bar{y}): X \rightrightarrows Y$ given by

$$
D F(\bar{x}, \bar{y})(x):=\{y \in Y \mid(x, y) \in C(\operatorname{graph}(F),(\bar{x}, \bar{y}))\} .
$$

Moreover, the second-order contingent derivative of $F$ at $(\bar{x}, \bar{y})$ in the direction $(u, v)$ is the set-valued map $D^{2} F(\bar{x}, \bar{y}, u, v): X \rightrightarrows Y$ defined by

$$
D^{2} F(\bar{x}, \bar{y}, u, v)(x):=\left\{y \in Y \mid(x, y) \in C^{2}(\operatorname{graph}(F),(\bar{x}, \bar{y}),(u, v))\right\} .
$$

The above derivatives have been used extensively in nonsmooth and variational analysis, viability theory, set-valued optimization, and numerous other related disciplines, see [7].

We have the following derivative characterization for the parameter-to-solution map:

Theorem 2.1. For $\ell \in A$, let $(u, p) \in \mathscr{U}(\ell)$ be given. Assume that the first-order contingent derivative $D \mathscr{U}(\ell, u, p): B \rightrightarrows V \times Q$ of the set-valued map $\mathscr{U}: A \rightrightarrows V \times Q$ at $(\ell, u, p) \in \operatorname{graph}(\mathscr{U})$ exists. Then for any direction $\delta \ell \in B$, any element $(\delta u, \delta p) \in D \mathscr{U}(\ell, u, p)(\delta \ell)$ satisfies the saddle point problem:

$$
\begin{aligned}
a(\ell, \delta u, v)+b(v, \delta p) & =-a(\delta \ell, u, v), \text { for every } v \in V, \\
b(\delta u, q) & =0, \text { for every } q \in Q .
\end{aligned}
$$

Proof. For the given $(\ell, u, p) \in \operatorname{graph}(\mathscr{U})$ and $\delta \ell \in B$, for any $(\delta u, \delta p) \in D \mathscr{U}(\ell, u, p)(\delta \ell)$, we have

$$
(\delta \ell, \delta u, \delta p) \in \operatorname{graph}(D \mathscr{U}(\ell, u, p))=C(\operatorname{graph}(\mathscr{U}),(\ell, u, p)),
$$

and by the definition of the contingent cone, there are sequences $\left\{t_{n}\right\} \subset \mathbb{P}$ and $\left\{\left(\ell_{n}, u_{n}, p_{n}\right)\right\}$ with $t_{n} \rightarrow 0$ and $\left(\ell_{n}, u_{n}, p_{n}\right) \rightarrow(\delta \ell, \delta u, \delta p)$ such that

$$
\left(\ell+t_{n} \ell_{n}, u+t_{n} u_{n}, p+t_{n} p_{n}\right) \in \operatorname{graph}(\mathscr{U}),
$$

or equivalently

$$
\left(u+t_{n} u_{n}, p+t_{n} p_{n}\right) \in \mathscr{U}\left(\ell+t_{n} \ell_{n}\right) .
$$


Therefore, by using the definition of the solution map $\mathscr{U}: A \rightrightarrows V \times Q$, we obtain

$$
\begin{aligned}
a\left(\ell+t_{n} \ell_{n}, u+t_{n} u_{n}, v\right)+b\left(v, p+t_{n} p_{n}\right) & =f(v), \text { for every } v \in V, \\
b\left(u+t_{n} u_{n}, q\right) & =g(q), \text { for every } q \in Q,
\end{aligned}
$$

which, after a simplification, implies that, for every $v \in V$ and for every $q \in Q$,

$$
\begin{aligned}
a(\ell, u, v)+t_{n} a\left(\ell, u_{n}, v\right)+t_{n} a\left(\ell_{n}, u, v\right)+t_{n}^{2} a\left(\ell_{n}, u_{n}, v\right)+b(v, p)+t_{n} b\left(v, p_{n}\right) & =f(v), \\
b(u, q)+t_{n} b\left(u_{n}, q\right) & =g(q),
\end{aligned}
$$

and by noting that the condition $(\ell, u, p) \in \operatorname{graph}(\mathscr{U})$ yields

$$
\begin{aligned}
a(\ell, u, v)+b(v, p) & =f(v), \text { for every } v \in V, \\
b(u, q) & =g(q), \text { for every } q \in Q,
\end{aligned}
$$

we obtain that the following system of equations hold

$$
\begin{array}{r}
a\left(\ell, u_{n}, v\right)+a\left(\ell_{n}, u, v\right)+t_{n} a\left(\ell_{n}, u_{n}, v\right)+b\left(v, p_{n}\right)=0, \text { for every } v \in V, \\
b\left(u_{n}, q\right)=0, \text { for every } q \in Q .
\end{array}
$$

By passing the above equations to the limit $n \rightarrow \infty$, we obtain

$$
\begin{aligned}
a(\ell, \delta u, v)+b(v, \delta p) & =-a(\delta \ell, u, v), \text { for every } v \in V, \\
b(\delta u, q) & =0, \text { for every } q \in Q
\end{aligned}
$$

and the desired identity (2.1) is proved. The proof is complete.

The following is the characterization of the second-order contingent derivative:

Theorem 2.2. For any $\ell \in A$, let $(u, p) \in \mathscr{U}(\ell)$ be given. Assume that second-order contingent derivative of the map $\mathscr{U}: A \rightrightarrows V \times Q$ at $(\ell, u, p) \in \operatorname{graph}(\mathscr{U})$ in a direction $(\delta \ell, \delta u, \delta p) \in$ $\operatorname{graph}(D \mathscr{U}(\ell, u, p))$ exists. Then for any $\delta^{2} \ell \in B$, any element

$$
\left(\delta^{2} u, \delta^{2} p\right) \in D^{2} \mathscr{U}(\ell, u, p, \delta \ell, \delta u, \delta p)\left(\delta^{2} \ell\right)
$$

satisfies the saddle point problem:

$$
\begin{aligned}
a\left(\ell, \delta^{2} u, v\right)+b\left(v, \delta^{2} p\right) & =-2 a(\delta \ell, \delta u, v)-a\left(\delta^{2} \ell, u, v\right), \text { for every } v \in V, \\
b\left(\delta^{2} u, q\right) & =0, \text { for every } q \in Q .
\end{aligned}
$$

Proof. For the given $(\ell, u, p) \in \operatorname{graph}(\mathscr{U})$ and the given $(\delta \ell, \delta u, \delta p) \in \operatorname{graph}(D \mathscr{U}(\ell, u, p))$, we assume that $\left(\delta^{2} u, \delta^{2} p\right) \in D^{2} \mathscr{U}(\ell, u, p, \delta \ell, \delta u, \delta p)\left(\delta^{2} \ell\right)$ is arbitrarily chosen. Then,

$$
\left(\delta^{2} \ell, \delta^{2} u, \delta^{2} p\right) \in \operatorname{graph}\left(D^{2} \mathscr{U}(\ell, u, p, \delta \ell, \delta u, \delta p)\right)=C^{2}(\operatorname{graph}(\mathscr{U}), \ell, u, p, \delta \ell, \delta u, \delta p) .
$$

Consequently, there are sequences $\left\{t_{n}\right\} \subset \mathbb{P}$ and $\left\{\left(\ell_{n}, u_{n}, p_{n}\right)\right\} \subset \operatorname{graph}(\mathscr{U})$ with $t_{n} \rightarrow 0$, and $\left(\ell_{n}, u_{n}, p_{n}\right) \rightarrow\left(\delta^{2} \ell, \delta^{2} u, \delta^{2} p\right)$ such that

$$
\left(\ell+t_{n} \delta \ell+\frac{1}{2} t_{n}^{2} \ell_{n}, u+t_{n} \delta u+\frac{1}{2} t_{n}^{2} u_{n}, p+t_{n} \delta p+\frac{1}{2} t_{n}^{2} p_{n}\right) \in \operatorname{graph}(\mathscr{U}) .
$$


This, due to the definition of the parameter-to-solution map $\mathscr{U}$, implies that

$$
\begin{aligned}
a\left(\ell+t_{n} \delta \ell+\frac{1}{2} t_{n}^{2} \ell_{n}, u+t_{n} \delta u+\frac{1}{2} t_{n}^{2} u_{n}, v\right)+b\left(v, p+t_{n} \delta p+\frac{1}{2} t_{n}^{2} p_{n}\right) & =f(v), \text { for every } v \in V \\
b\left(u+t_{n} \delta u+\frac{1}{2} t_{n}^{2} u_{n}, q\right) & =g(q), \text { for every } q \in Q,
\end{aligned}
$$

which we simplify to deduce that, for every $v \in V$ and for every $q \in Q$,

$$
\begin{array}{r}
a(\ell, u, v)+t_{n} a(\ell, \delta u, v)+\frac{1}{2} t_{n}^{2} a\left(\ell, u_{n}, v\right)+t_{n} a(\delta \ell, u, v)+t_{n}^{2} a(\delta \ell, \delta u, v)+\frac{1}{2} t_{n}^{3} a\left(\delta \ell, u_{n}, v\right)+ \\
\frac{1}{2} t_{n}^{2} a\left(\ell_{n}, u, v\right)+\frac{1}{2} t_{n}^{3} a\left(\ell_{n}, \delta u, v\right)+\frac{1}{4} t_{n}^{4} a\left(\ell_{n}, u_{n}, v\right)+b(v, p)+t_{n} b(v, \delta p)+\frac{t_{n}^{2}}{2} b\left(v, p_{n}\right)=f(v), \\
b(u, q)+t_{n} b(\delta u, q)+\frac{t_{n}^{2}}{2} b\left(u_{n}, q\right)=g(q) .
\end{array}
$$

To the above system, we first apply the fact that $(\ell, u, p) \in \operatorname{graph}(\mathscr{U})$, and then divide both sides of the resulting identities by $t_{n}$, to obtain the following system

$$
\begin{aligned}
a(\ell, \delta u, v)+\frac{1}{2} t_{n} a\left(\ell, u_{n}, v\right)+a(\delta \ell, u, v)+t_{n} a(\delta \ell, \delta u, v)+\frac{1}{2} t_{n}^{2} a\left(\delta \ell, u_{n}, v\right) & \\
\frac{1}{2} t_{n} a\left(\ell_{n}, u, v\right)+\frac{1}{2} t_{n}^{2} a\left(\ell_{n}, \delta u, v\right)+\frac{1}{4} t_{n}^{3} a\left(\ell_{n}, u_{n}, v\right)+b(v, \delta p)+\frac{t_{n}}{2} b\left(v, p_{n}\right) & =0, \text { for every } v \in V, \\
b(\delta u, q)+\frac{t_{n}}{2} b\left(u_{n}, q\right) & =0, \text { for every } q \in Q .
\end{aligned}
$$

We now use the fact that $(\delta \ell, \delta u, \delta p) \in \operatorname{graph}(D \mathscr{U}(\ell, u, p))$, and then divide both sides of the resulting identities by $t_{n}$ to obtain

$$
\begin{aligned}
\frac{1}{2} a\left(\ell, u_{n}, v\right)+a(\delta \ell, \delta u, v)+\frac{1}{2} t_{n} a\left(\delta \ell, u_{n}, v\right)+\frac{1}{2} a\left(\ell_{n}, u, v\right)+\frac{1}{2} t_{n} a\left(\ell_{n}, \delta u, v\right) & \\
\frac{1}{4} t_{n}^{2} a\left(\ell_{n}, u_{n}, v\right)+\frac{1}{2} b\left(v, p_{n}\right) & =0, \text { for every } v \in V \\
\frac{1}{2} b\left(u_{n}, q\right) & =0, \text { for every } q \in Q .
\end{aligned}
$$

We pass the above system to the limit $t_{n} \rightarrow 0$ and obtain

$$
\begin{aligned}
a\left(\ell, \delta^{2} u, v\right)+b\left(v, \delta^{2} p\right) & =-2 a(\delta \ell, \delta u, v)-a\left(\delta^{2} \ell, u, v\right), \text { for every } v \in V, \\
b\left(\delta^{2} u, q\right) & =0, \text { for every } q \in Q
\end{aligned}
$$

which proves (2.2). The proof is complete.

Regularization methods have been used extensively in the context of saddle point problems, see $[4,5]$. To describe the regularization, we consider the setting where the data set of the saddle point problem is contaminated by some noise in the sense described below. Let $\left\{\varepsilon_{n}\right\}$, and $\left\{v_{n}\right\}$ be sequences of positive reals. For each $n \in \mathbb{N}$, let $f_{v_{n}} \in V^{*}, g_{v_{n}} \in Q^{*}$, be such that

$$
\max \left\{\left\|f_{v_{n}}-f\right\|_{V^{*}},\left\|g_{v_{n}}-g\right\|_{Q^{*}}\right\} \leq v_{n},
$$

Moreover, as $n \rightarrow \infty$, the sequences $\left\{\varepsilon_{n}\right\}$ and $\left\{v_{n}\right\}$ satisfy

$$
\left\{\varepsilon_{n}, v_{n}, \frac{v_{n}}{\varepsilon_{n}}\right\} \rightarrow 0 \text {. }
$$


We consider the following family of regularized saddle point problems: For $n \in \mathbb{N}$, given the regularization parameter $\varepsilon_{n}>0$ and $\ell \in A$, find $\left(u_{n}, p_{n}\right) \equiv\left(u_{n}(\ell), p_{n}(\ell)\right) \in V \times Q$ such that

$$
\begin{aligned}
a\left(\ell, u_{n}, v\right)+\varepsilon_{n}\left\langle u_{n}, v\right\rangle_{V}+b\left(v, p_{n}\right) & =f_{v_{n}}(v), \text { for every } v \in V, \\
b\left(u_{n}, q\right)-\varepsilon_{n}\left\langle p_{n}, q\right\rangle_{Q} & =g_{v_{n}}(q), \text { for every } q \in Q .
\end{aligned}
$$

As a direct consequence of the Lax-Milgram lemma, for fixed $n \in \mathbb{N}$ and $\ell \in A$, the regularized saddle point problem (2.5) has a unique solution $\left(u_{n}(\ell), p_{n}(\ell)\right)$. Therefore, the regularized parameter-to-solution map $\ell \rightarrow\left(u_{n}(\ell), p_{n}(\ell)\right)$ is well-defined and single-valued. The following result sheds some light on the smoothness of the regularized parameter-to-solution map:

Theorem 2.3. Let $\ell$ be in the interior of $A$. For a fixed element $n \in \mathbb{N}$, the first-order derivative $\left(D u_{n}(\ell) \delta \ell, D p_{n}(\ell) \delta \ell\right)$ of the regularized parameter-to-solution map $\ell \rightarrow\left(u_{n}(\ell), p_{n}(\ell)\right)$ at $\ell$ in the direction $\delta \ell \in B$ is the unique solution of the following regularized saddle point problem with the regularization parameter $\varepsilon_{n}>0$ :

$$
\begin{aligned}
a\left(\ell, D u_{n}(\ell) \delta \ell, v\right)+ & \varepsilon_{n}\left\langle D u_{n}(\ell) \delta \ell, v\right\rangle_{V}+b\left(v, D p_{n}(\ell) \delta \ell\right)=-a\left(\delta \ell, u_{n}(\ell), v\right), \text { for every } v \in V \\
b\left(D u_{n}(\ell) \delta \ell, q\right)-\varepsilon_{n}\left\langle D p_{n}(\ell) \delta \ell, q\right\rangle_{Q} & =0, \text { for every } q \in Q
\end{aligned}
$$

Moreover, the second-order derivative $\left(D^{2} u_{n}(\ell)\left(\delta \ell_{1}, \delta \ell_{2}\right), D^{2} p_{n}(\ell)\left(\delta \ell_{1}, \delta \ell_{2}\right)\right)$ of $\left(u_{n}(\ell), p_{n}(\ell)\right)$ at $\ell$ in the direction $\left(\delta \ell_{1}, \delta \ell_{2}\right) \in B \times B$ is the unique solution of the following regularized saddle point problem:

$$
\begin{aligned}
a\left(\ell, D^{2} u_{n}(\ell)\left(\boldsymbol{\delta} \ell_{1}, \delta \ell_{2}\right), v\right) & +\varepsilon_{n}\left\langle D^{2} u_{n}(\ell)\left(\boldsymbol{\delta} \ell_{1}, \boldsymbol{\delta} \ell_{2}\right), v\right\rangle_{V}+b\left(v, D^{2} p_{n}(\ell)\left(\boldsymbol{\delta} \ell_{1}, \boldsymbol{\delta} \ell_{2}\right)\right) \\
& =-a\left(\boldsymbol{\delta} \ell_{2}, D u_{n}(\ell) \boldsymbol{\delta} \ell_{1}, v\right)-a\left(\boldsymbol{\delta} \ell_{1}, D u_{n}(\ell) \boldsymbol{\delta} \ell_{2}, v\right), \text { for every } v \in V
\end{aligned}
$$

$$
b\left(D^{2} u_{n}(\ell)\left(\delta \ell_{1}, \delta \ell_{2}\right), q\right)-\varepsilon_{n}\left\langle D^{2} p_{n}(\ell)\left(\delta \ell_{1}, \delta \ell_{2}\right), q\right\rangle_{Q}=0, \text { for every } q \in Q
$$

Proof. The proof follows by similar arguments that were used in [6].

We give necessary conditions under which the derivative of the regularized parameter-tosolution map remains bounded.

Theorem 2.4. For a parameter $\ell$ in the interior of $A$, let $(u, p) \in \mathscr{U}(\ell)$ be a given point. Assume that the first-order contingent derivative of the set-valued parameter-to-solution map $\mathscr{U}: A \rightrightarrows$ $V \times Q$ at the point $(\ell, u, p) \in \operatorname{graph}(\mathscr{U})$ exists. If,

$$
\left\|u_{n}(\ell)-u\right\|_{V}=O\left(\varepsilon_{n}\right)
$$

where $\left(u_{n}(\ell), p_{n}(\ell)\right)$ is the regularized solution of (2.5) for parameter $\ell$, then the first-order derivative $\left(D u_{n}(\ell) \boldsymbol{\delta} \ell, D p_{n}(\ell) \boldsymbol{\delta} \ell\right)$ of $\left(u_{n}(\ell), p_{n}(\ell)\right)$ in any direction $\boldsymbol{\delta} \ell \in B$ is uniformly bounded.

Proof. From Theorem 2.1, for any $\delta \ell \in B$, and any $(\delta u, \delta p) \in D \mathscr{U}(\ell, u, p)(\delta \ell)$, we have

$$
\begin{aligned}
a(\ell, \delta u, v)+b(v, \delta p) & =-a(\delta \ell, u, v), \text { for every } v \in V, \\
b(\delta u, q) & =0, \text { for every } q \in Q .
\end{aligned}
$$

Due to Theorem 2.3, for a fixed $n \in \mathbb{N}$ and the regularization parameter $\varepsilon_{n}>0$, the first-order derivative $\left.\left(\delta u_{n}, \delta p_{n}\right):=\left(D u_{n}(\ell) \delta \ell, D p_{n}(\ell) \delta \ell\right)\right)$ of the map $\ell \rightarrow\left(u_{n}(\ell), p_{n}(\ell)\right)$ at $\ell$ in the direction 
$\delta \ell \in B$ is characterized by the regularized saddle point problem:

$$
\begin{aligned}
a\left(\ell, \delta u_{n}, v\right)+\varepsilon_{n}\left\langle\delta u_{n}, v\right\rangle_{V}+b\left(v, \delta p_{n}\right) & =-a\left(\delta \ell, u_{n}, v\right), \quad \text { for every } v \in V, \\
b\left(\delta u_{n}, q\right)-\varepsilon_{n}\left\langle\delta p_{n}, q\right\rangle_{Q} & =0, \quad \text { for every } q \in Q .
\end{aligned}
$$

We subtract (2.9) from (2.10) and rearrange the resulting equations to obtain

$$
\begin{aligned}
a\left(\ell, \delta u_{n}-\delta u, v\right)+\varepsilon_{n}\left\langle\delta u_{n}, v\right\rangle_{V}+b\left(v, \delta p_{n}-\delta p\right)=a\left(\delta \ell, u-u_{n}, v\right), & \text { for every } v \in V, \\
b\left(\delta u_{n}-\delta u, q\right)-\varepsilon_{n}\left\langle\delta p_{n}, q\right\rangle_{Q}=0, & \text { for every } q \in Q .
\end{aligned}
$$

We set $v=\delta u_{n}-\delta u, q=\delta p_{n}-\delta p$, and combine the resulting equations to obtain

$$
\begin{aligned}
& \varepsilon_{n}\left\|\delta u_{n}-\delta u\right\|_{V}^{2}+\varepsilon_{n}\left\|\delta p_{n}-\delta p\right\|_{Q}^{2} \\
& \leq a\left(\ell, \delta u_{n}-\delta u, \delta u_{n}-\delta u\right)+\varepsilon_{n}\left\langle\delta u_{n}-\delta u, \delta u_{n}-\delta u\right\rangle_{V}+\varepsilon_{n}\left\langle\delta p_{n}-\delta p, \delta p_{n}-\delta p\right\rangle_{Q} \\
& =a\left(\delta \ell, u-u_{n}, \delta u_{n}-\delta u\right)-\varepsilon_{n}\left\langle\delta u, \delta u_{n}-\delta u\right\rangle_{V}-\varepsilon_{n}\left\langle\delta p, \delta p_{n}-\delta p\right\rangle_{Q},
\end{aligned}
$$

which further implies that

$$
\begin{aligned}
& \left\|\delta u_{n}-\delta u\right\|_{V}^{2}+\left\|\delta p_{n}-\delta p\right\|_{Q}^{2} \\
& \leq \varsigma_{0}\|\delta \ell\|_{B}\left\|u_{n}-u\right\|_{V} \frac{\left\|\delta u_{n}-\delta u\right\|_{V}}{\varepsilon_{n}}+\|\delta u\|_{V}\left\|\delta u_{n}-\delta u\right\|_{V}+\|\delta p\|_{Q}\left\|\delta p_{n}-\delta p\right\|_{Q},
\end{aligned}
$$

(with $\varsigma_{0}>0$ from assumption (1.1c)) and the conclusion follows at once. The proof is complete.

\section{CONCLUding REMARKS}

We presented characterizations of the first-order and the second-order contingent derivatives of the set-valued parameter-to-solution map under the critical assumption that these derivatives exist. This is quite a natural step as we are working under the assumption that the saddle point problem has a non-empty solution set for each parameter. Since we know that the saddle point problem is uniquely solvable under the Inf-Sup condition, a possible extension of the above results is to obtain the derivative characterization under the Inf-Sup condition and compare those derivatives with the ones given above.

The elliptic regularization for variational problems, under the assumption that the original problem is solvable, combines the variational problem with the regularized variational problem to deduce that the regularized solutions are uniformly bounded (see $[3,8]$ ). The next step is to extract a subsequence that converges weakly to a solution of the original variational problem. An alternate view of this process is that any condition that proves that the regularized solutions are bounded turns out to be a sufficient condition for the solvability of the original variational problem. In Theorem 2.4, we employed this idea to prove the boundedness of the derivatives. In the present context, the role of the original variational problem is played by the derivative characterization involving the first-order contingent derivative. As a consequence, suitable conditions ensuring the boundedness of the derivatives of the regularized parameter-to-solution map can be used to show the contingent differentiability of the parameter-to-solution map.

\section{Acknowledgements}

The research of Baasansuren Jadamba and Akhtar Khan was supported by the National Science Foundation under the Award No.1720067. Miguel Sama is supported by Ministerio de 
Ciencia, Innovación y Universidades (MCIU), Agencia Estatal de Investigación (AEI) (Spain) and Fondo Europeo de Desarrollo Regional (FEDER) under project PGC2018-096899-B-I00 (MCIU/AEI/FEDER, UE) and [grant number 2020-MAT11] (ETSI Industriales, UNED).

\section{REFERENCES}

[1] P. B. Bochev, M. D. Gunzburger, R. B. Lehoucq, On stabilized finite element methods for the Stokes problem in the small time step limit, Internat. J. Numer. Methods Fluids 53 (2007), 573-597.

[2] P. B. Bochev, R. B. Lehoucq, Regularization and stabilization of discrete saddle-point variational problems, Electron. Trans. Numer. Anal. 22 (2006), 97-113.

[3] F. Giannessi, A. A. Khan, Regularization of non-coercive quasi variational inequalities, Control Cybernet. 29 (2000) 91-110.

[4] V. Girault, P.-A. Raviart, Finite element approximation of the Navier-Stokes equations, vol. 749 of Lecture Notes in Mathematics, Springer-Verlag, Berlin-New York, 1979.

[5] K. Ito, K. Kunisch, G. Peichl, On the regularization and approximation of saddle point problems without inf-sup condition, Comput. Appl. Math. 21 (2002), 245-274.

[6] B. Jadamba, A. A. Khan, G. Rus, M. Sama, B. Winkler, A new convex inversion framework for parameter identification in saddle point problems with an application to the elasticity imaging inverse problem of predicting tumor location, SIAM J. Appl. Math. 74 (2014), 1486-1510.

[7] A. A. Khan, C. Tammer, C. Zalinescu, Regularization of quasi-variational inequalities, Optimization 64 (2015), 1703-1724.

[8] A. A. Khan, C. Tammer, C. Zalinescu, Set-valued optimization, Springer, Heidelberg, 2015.

[9] B. S. Mordukhovich, Variational analysis and applications, Springer Monographs in Mathematics, Springer, Cham, 2018. 